\title{
Clinical course of thyroid function and thyroid associated-ophthalmopathy in patients with euthyroid Graves' disease
}

This article was published in the following Dove Press journal:

Clinical Ophthalmology

\author{
Nami Suzuki' \\ Jaeduk Yoshimura Noh' \\ Toshiaki Kameda' \\ Ai Yoshihara' \\ Hidemi Ohye' \\ Miho Suzuki' \\ Masako Matsumoto' \\ Yo Kunii' \\ Kenji Iwaku' \\ Natsuko Watanabe' \\ Koji Mukasa' \\ Ai Kozaki² \\ Toshu Inoue ${ }^{2}$ \\ Kiminori Sugino' \\ Koichi Ito' \\ 'Department of Internal Medicine, Ito \\ Hospital, Tokyo, Japan; ${ }^{2}$ Department of \\ Ophthalmology, Olympia Eye Hospital, \\ Tokyo, Japan
}

Background: Euthyroid Graves' disease (EGD) is a rare condition defined as the presence of thyroid-associated ophthalmopathy (TAO) in patients with normal thyroid function. Due to the rarity of this disease, only a limited number of studies and case reports are available for further evaluation of the characteristics of the disease. The aim of this study was to examine the changes in the thyroid function, thyrotropin receptor antibodies (TRAb) and eye symptoms, and then determine whether TRAb is related to TAO in EGD patients. TRAb in this study was defined as including both thyrotropin-binding inhibitory immunoglobulin (TBII) and thyroidstimulating immunoglobulin (TSAb).

Patients and methods: Medical records of patients diagnosed with EGD were reviewed. Ophthalmologists specializing in TAO examined the eyes of all subjects.

Results: Of the 58 patients diagnosed with EGD, 24.1\% developed hyperthyroidism, while 3.4\% developed hypothyroidism. A total of $72.4 \%$ of the 58 patients remained euthyroid throughout the entire follow-up period. At the initial presentation, TBII and TSAb were positive in $74.5 \%$ and $70.5 \%$, respectively. Ophthalmic treatments were administered to $30(51.7 \%)$ out of the 58 patients. A significant spontaneous improvement of the eye symptoms was found in 28 of the EGD patients who did not require eye treatments. EGD patients exhibited positive rates for both TBII and TSAb, with the number of the TRAb-positive patients gradually decreasing while the eye symptoms spontaneously improved over time. There were no correlations found between TRAb at initial presentation and the eye symptoms.

Conclusion: TBII and TSAb were positive in about $70 \%$ of EGD patients at their initial visit. Thyroid functions of EGD patients who have been euthyroid for more than 6.7 years may continue to remain euthyroid in the future.

Keywords: euthyroid Graves' orbitopathy, euthyroid Graves' ophthalmopathy, thyroid-associated ophthalmopathy, TSH receptor antibody, thyroid-stimulating immunoglobulin

\section{Introduction}

Euthyroid Graves' disease (EGD) is a rare condition that is defined as the presence of thyroid-associated ophthalmopathy (TAO) in patients having normal thyroid function without a prior history of treatment. EGD was first reported in 1945 by Rundle and Wilson, ${ }^{1}$ who examined and followed 2 patients for less than 30 months. In general, TAO is found in patients with hyperthyroid Graves' disease (GD). A previous study reported that the onset of TAO preceded thyroid dysfunction in $19.6 \%$ of the GD patients, ${ }^{2}$ while another study reported that TAO occurred within 18 months prior to or followed by the onset of thyroid dysfunction in about $85 \%$ of the GD patients. ${ }^{3}$ Among the TAO patients, the prevalence of EGD has been reported to range from
Correspondence: Nami Suzuki Department of Internal Medicine, Ito Hospital, 4-3-6 Jingumae, Shibuya-ku, Tokyo 150-8308, Japan

$\mathrm{Tel}+8 \mathrm{I} 334027447$

Fax +81334027439

Email n-suzuki@ito-hospital.jp 
$0.7 \%$ to $21 \% .^{2,4,5}$ Due to the rarity of this disease, there are still only a limited number of studies and case reports that can be used to further evaluate the characteristics of the disease. Moreover, TAO is said to be related to the thyrotropin receptor antibody (TRAb), which includes thyroid-stimulating immunoglobulin (TSAb) and thyrotropin-binding inhibitory immunoglobulin (TBII). Khoo et $\mathrm{al}^{5}$ reported finding positive ratios for the TRAb in 6 EGD patients when using four different measurement methods, with TRAb detected by at least one of the methods in all 6 patients. On the other hand, Tabasum et $\mathrm{al}^{6}$ reported finding an EGD case with a negative TRAb at the time of the development of TAO. While it is possible that these variations might be due to differences in the measurement methods, the relationship between TRAb and the severity of TAO in EGD patients has yet to be definitively established. Therefore, the aim of this study was to examine the changes in thyroid function, anti-thyroid antibody and eye symptoms, in addition to determining the relationship between TRAb and TAO in EGD patients.

\section{Patients and methods}

\section{Patients}

This retrospective study enrolled subjects who had visited both Ito Hospital, which is a hospital that specializes in thyroid disease, and Olympia Eye Hospital, which is a hospital that specializes in TAO, between March 1987 and July 2013, and were diagnosed as EGD on their initial presentation. Since both thyroid function and eye observations were equally essential for accomplishing the overall aims of this study, patients who lacked complete data were excluded from our analyses. All the subjects were followed up for at least 6 months. Serum levels of $\mathrm{FT}_{3}, \mathrm{FT}_{4}$ and thyroid-stimulating hormone (TSH) were measured in all patients, with some patients also undergoing measurements for TBII and TSAb. In the EGD patients who were initially examined after 2006 , ultrasonography was used to estimate the thyroid volume. EGD was diagnosed based on both normal thyroid function ( $\mathrm{FT}_{3}, \mathrm{FT}_{4}$ and $\mathrm{TSH}$ levels), which was determined at the first visit, and evidence of TAO. Ophthalmologists specializing in TAO diagnosed all the TAO patients. Ophthalmic symptoms used to determine TAO were based on the critera established by the Japanese Thyroid Association, which includes eyelid fissure enlargement ( $>8 \mathrm{~mm}$ on each side), proptosis (Hertel measurement $>17 \mathrm{~mm}$ on each side), optic nerve dysfunction, corneal or conjunctival involvement and orbital involvement determined by computed tomography (CT) or magnetic resonance imaging (MRI) in order to evaluate the extraocular muscle involvement. ${ }^{7}$ To measure the cross-sectional areas of the rectus muscles, we used the MRI coronal view and outlined each of the extraocular muscles for use in calculating the cross-sectional area. Subsequently, we then added up the total areas of the 8 muscles. In addition to confirming the evidence of TAO, CT and MRI were also used to exclude any orbital space-occupying lesion that could be responsible for the ophthalmic dysfunction, such as tumor or extraocular myositis. ${ }^{8}$ Since the EGD diagnosis was made based on an exclusion diagnosis, the use of CT and MRI helped ensure the accuracy of the diagnosis. Based on this definition, 58 patients were diagnosed with EGD at their first visit. The medical records of all subjects were retrospectively examined.

The measured length, width and thickness of the thyroid ultrasonogram were used to calculate the estimated thyroid volume. The formula used to calculate the volume was as follows: Volume $=(0.7365 \times$ right lobe length $\times$ width $\times$ thickness $+0.7412 \times$ left lobe length $\times$ width $\times$ thickness) $-0.55{ }^{9}$

The study was approved by the Ethics Committee of Ito Hospital. As this study analyzed the existing medical information without accompanying patient's invasion, a written informed consent was waived by the approving ethics committee, in accordance with Japanese Ethical Guidelines for Medical and Health Research Involving Human Subjects.

All the data acquired from medical record were anonymized, and therefore, the patient confidentiality was protected.

\section{Laboratory methods TBII}

TBII levels were measured using a radioimmunoassay kit until September 2008. A TRAb kit (Baxter, Deerfield, IL, USA) was initially used starting in 1982 and continued until September 1992. Subsequent TRAb kits used included the TRAb kit (Cosmic, Tokyo, Japan) between October 1992 and May 1995, the TRAb II kit (Cosmic) between June 1995 and January 1999, the TRAb III kit (Cosmic) between February 1999 and June 2003 and the TRAb CT (Cosmic) between July 2003 and September 2008. Levels are expressed in terms of the inhibition index of the TSH binding and are presented as percentages (the $<10 \%$ reference range was defined by the manufacturer). Starting from October 2008, all levels were measured using an ECLusys TRAb electrochemiluminescence immunoassay (normal range: $<2.0 \mathrm{IU} / \mathrm{L}$; Roche Diagnostics, Basel, Switzerland). A formula derived from the regression curve representing the relationship between the TRAb CT value and the ECLusys value was used to compare the two values. 


\section{TSAb}

TSAb was measured using a TSAb radioimmunoassay and bioassay kit (normal range $<180 \%$; Yamasa, Choshi, Japan) until 2014.

\section{Thyroid hormones and TSH assay}

$\mathrm{FT}_{3}, \mathrm{FT}_{4}$ and $\mathrm{TSH}$ levels were measured using kits that were commercially available at that time.

\section{Statistical analysis}

Data were calculated and analyzed using JMP version 11.0 software (SAS Institute Inc, Cary, NC, USA). A chi-squared test was used to test the significance of the differences between the groups, and a paired $t$-test was used to analyze the significance of the differences of the items in the same groups but at a different times. Spearman's rank correlation coefficient was used to analyze the correlation between the TRAb (TBII and TSAb) and the ophthalmic observations (proptosis, lid fissure and the sum of the area for the 8 ocular muscles) at the initial visit. Kruskal-Wallis test was used to analyze the significance of the differences between the multiple groups. Kaplan-Meier analysis was carried out to measure the time remaining for normal thyroid function in patients with EGD after their initial diagnosis.

\section{Results}

\section{Patient characteristics on initial presentation}

Data were collected from the medical records of 58 EGD patients ( 13 males and 45 females) with a mean age of 43 years (Table 1). The patients were followed up for a minimum of 7 months unless they developed thyroid dysfunction, with a median observation period of 5.3 (range 0.6-29.7) years. The smoking rate of the subjects was $20.7 \%$. At the initial presentation, available data showed that $74.5 \%$ of the $55 \mathrm{EGD}$ patients were positive for TBII, while $70.5 \%$ of 44 EGD patients were positive for TSAb. There were no significant differences between the positive rates of TBII and TSAb ( $p=0.8152$ ) (Table 1). The incidence of initial TBII evaluated by the Cosmic kit was $67.7 \%$ (21/31), while for the Roche kit, it was $80.0 \%(20 / 25)$. Due to the differences in the subjects, the precision of these 2 kits could not be directly compared.

Ophthalmologists examined all the 58 patients. The median values for the sum of the bilateral lid fissure, proptosis and cross-sectional areas of the 8 extraocular rectus muscles were $34 \mathrm{~mm}$ (range 19-49 mm), $18 \mathrm{~mm}$ (range 11-36 mm) and $2.43 \mathrm{~cm}^{2}$ (range $1.11-5.80 \mathrm{~cm}^{2}$ ), respectively. Since no significant laterality of eye symptoms was seen in any of the
Table I Characteristics of the 58 patients initially diagnosed with EGD

\begin{tabular}{|c|c|}
\hline Number of patients & 58 \\
\hline Sex & $\begin{array}{l}\text { Male: I3 (22.4\%); female: } 45 \\
(77.6 \%)\end{array}$ \\
\hline Age (years) & $43.6 \pm 13.3$ \\
\hline Observation period (years) & 5.8 (range 0.6-29.7) \\
\hline Smoking rate & $12 / 58(20.7 \%)$ \\
\hline Estimated thyroid volume $(\mathrm{mL}, \mathrm{n}=35)$ & I3.1 g (range 5.3-39.8) \\
\hline TBII (IU/L) (Cosmic:Roche =3I:25) & 2.5 (range $0.6-47.2$ ) \\
\hline Positive rate & $4 I / 55(74.5 \%)$ \\
\hline Using Cosmic & $21 / 31(67.7 \%)$ \\
\hline Using Roche & $20 / 25(80.0 \%)$ \\
\hline TSAb $(\%, n=44)$ & 257 (range II0-I,448) \\
\hline Positive rate & $3 \mathrm{l} / 44(70.5 \%)$ \\
\hline Sum of lid fissures* $(\mathrm{mm})$ & 34 (range 19-49) \\
\hline Proptosis* $(\mathrm{mm})$ & I8 (range II-36) \\
\hline Eight extraocular muscles* $\left(\mathrm{cm}^{2}\right)$ & 2.43 (range I.II-5.80) \\
\hline \multicolumn{2}{|l|}{ Course of thyroid function } \\
\hline Remained euthyroid & $\begin{array}{l}42(72.4 \%) \text {, male: } 7 \text {; female: } 35 \text {; } \\
\text { mean age, } 43.2 \pm 12.4 \text { years }\end{array}$ \\
\hline Developed hyperthyroidism & $\begin{array}{l}\text { I4 }(24.1 \%) \text {, male: } 6 \text {; female: } 8 \\
\text { mean age, } 42.9 \pm 16.9 \text { years }\end{array}$ \\
\hline Developed hypothyroidism & $\begin{array}{l}2(3.4 \%) \text {, male: } 0 \text {; female: } 2 \text {; } \\
\text { ages } 50 \text { and } 54 \text { years }\end{array}$ \\
\hline \multicolumn{2}{|c|}{ Duration until development of dysfunction (months) } \\
\hline Hyperthyroidism & 28.5 (range $3-124)$ \\
\hline Hypothyroidism & 25 and 39 \\
\hline
\end{tabular}

Notes: *Right + left. Numerical values are reported as the median, with the exception of age (mean \pm SD)

Abbreviations: EGD, euthyroid Graves' disease; TBIl, thyrotropin-binding inhibitory immunoglobulin; TSAb, thyroid-stimulating immunoglobulin.

patients in this study, the sums of the eyelid fissure, proptosis and cross-sectional area of the extraocular muscles were used for comparing the course of the eye symptoms.

Figure 1 shows the correlation between the TRAb (TBII and TSAb) and the ophthalmic observations (proptosis, lid fissure and the sum of the 8 ocular muscles) at the initial visit. The current study found no statistical correlation between the TRAb (TBII and TSAb) and the ophthalmic symptoms.

\section{Clinical course of thyroid function and anti-thyroid antibodies}

During the follow-up, $14(24.1 \%)$ out of the 58 patients subsequently developed hyperthyroidism, while $2(3.4 \%)$ out of the 58 patients developed hypothyroidism. The median period until developing hyperthyroidism was $2.4(0.3-10.3)$ years. With the exception of the 2 cases with hyperthyroidism, the thyroid dysfunction occurred within 6.7 years in these patients. The hyperthyroidism that developed in the 2 patients occurred at 9.4 years and 10.3 years after the TAO diagnosis. Throughout the follow-up period, 42 (72.4\%) out of the 58 patients remained euthyroid. Figure 2 presents the 

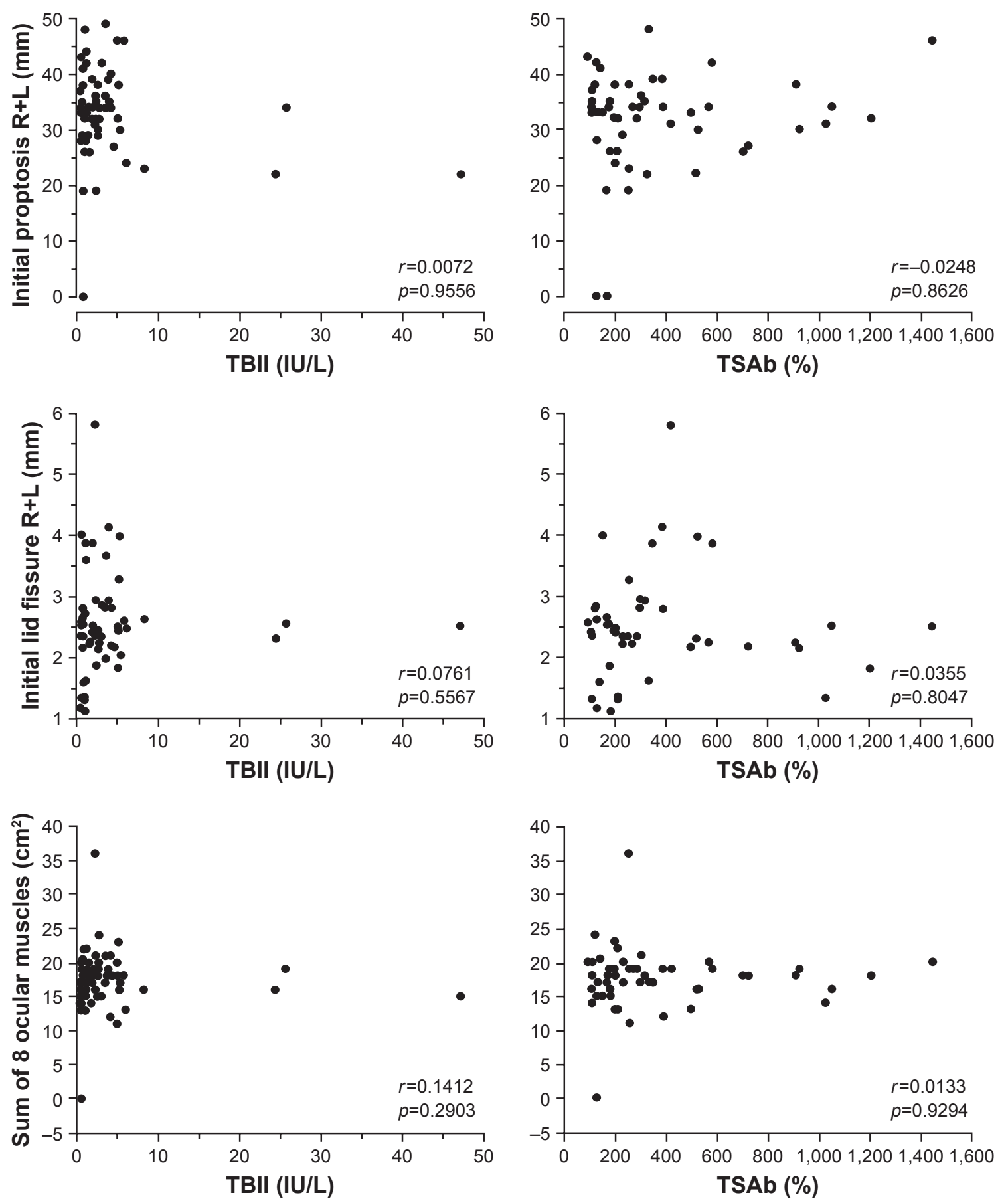

Figure I Correlation between TRAb (TBIl and TSAb) and eye symptoms.

Notes: Correlation between TRAb (TBII and TSAb) and ophthalmic observations (proptosis, lid fissure and sum of 8 ocular muscles) on the initial visit were analyzed using Spearman's rank correlation coefficients. No statistical correlation was observed between the TRAb and eye symptoms.

Abbreviations: TRAb, thyrotropin receptor antibodies; TBIl, thyrotropin-binding inhibitory immunoglobulin; TSAb, thyroid-stimulating immunoglobulin; L, left; R, right.

Kaplan-Meier curve that describes the time of remaining for normal thyroid function in the patients with EGD after their initial diagnosis.

Figure 3 shows the clinical course of the positive rates of the TBII and TSAb in the EGD patients who remained euthyroid. The number of the patients who were positive for TBII or TSAb gradually decreased spontaneously over time, with none of the patients showing any increase in either TBII or TSAb during the observation period. On the other hand, the TBII values for the patients who subsequently developed hyperthyroidism were available in 12 out of 14 patients, with an increased TBII value seen in 11 out of these 12 patients. The TBII values in the remaining 2 patients were not measured. 


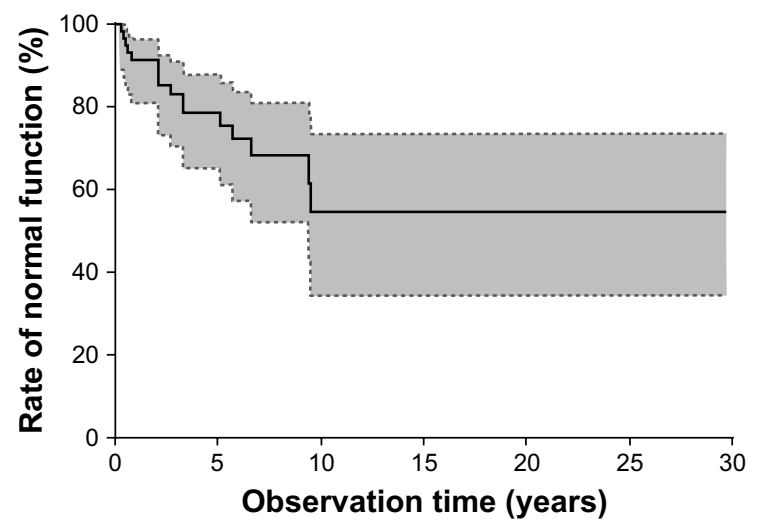

Figure 2 Kaplan-Meier curve describing the time remaining for normal thyroid function in 58 EGD patients.

Notes: Thyroid dysfunction occurred in 16 out of 58 EGD patients. Hyperthyroidism developed in 14 and hypothyroidism developed in 2 patients. Out of the 58 patients, 42 remained euthyroid. With the exception of 2 cases, thyroid dysfunction occurred within 6.7 years after the development of ophthalmopathy. The gray area shows the $95 \%$ confidence interval.

Abbreviation: EGD, euthyroid Graves' disease.

\section{Clinical course of the ophthalmopathy}

Based on the initial diagnosis of TAO, 29 (50.0\%) out of 58 patients received ophthalmic treatments that included local injection of triamcinolone, oral steroid therapy, radiotherapy and surgery (Table 2). Of these, local injection was the most frequently chosen treatment in $62.0 \%(18 / 29)$, with oral

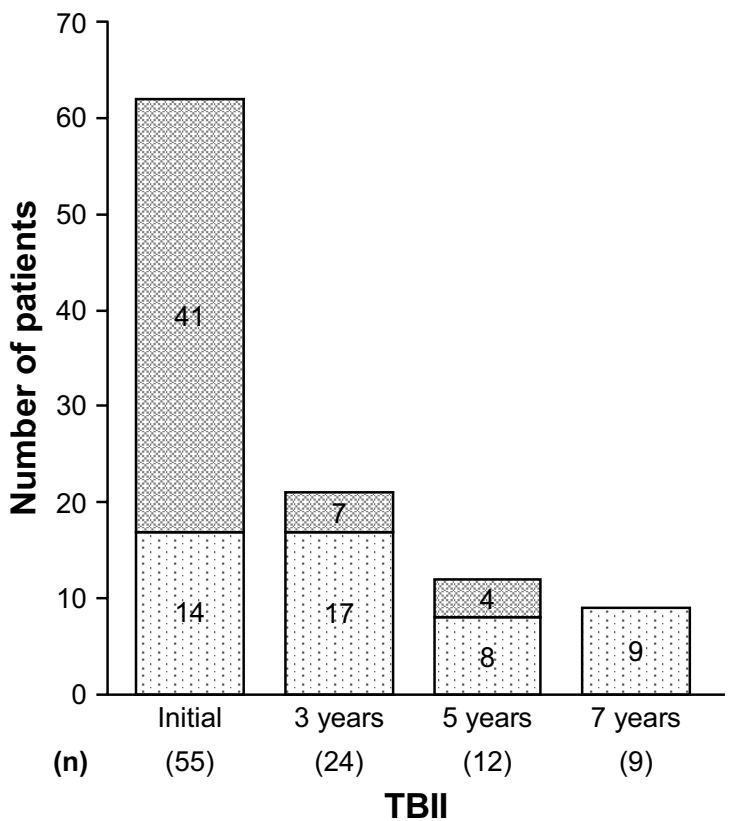

TBII steroid therapy given to 2 patients. Radiation was administered in $55.2 \%(16 / 29)$ of the patients, while $20.6 \%(6 / 29)$ underwent surgery (with all 6 of these patients undergoing extraocular muscle surgery and 1 patient additionally undergoing orbital decompression surgery). Among the 16 patients who received radiation, it was administered as monotherapy in 8 patients, as they had mild TAO and the potential adverse reaction associated with intravenous glucocorticoid therapy did not seem to outweigh the therapeutic effect. ${ }^{10}$ Combined therapy was administered in 9 of these 29 patients at the initial visit, with 11 out of 30 patients being eventually administered this therapy during the overall study period. While in most cases, the TAO symptoms improved and stabilized with or without treatment over time, there were 3 patients who subsequently relapsed a few years later and required additional treatments. The additional therapies administered to the patients included intravenous methylprednisolone, local injection of steroid and surgery. Although one of these patients did not require any treatment at the initial visit, intravenous methylprednisolone therapy was subsequently required 2 years after the development of EGD.

The eye symptoms of the 29 EGD patients who did not need any eye treatments at their initial presentation showed

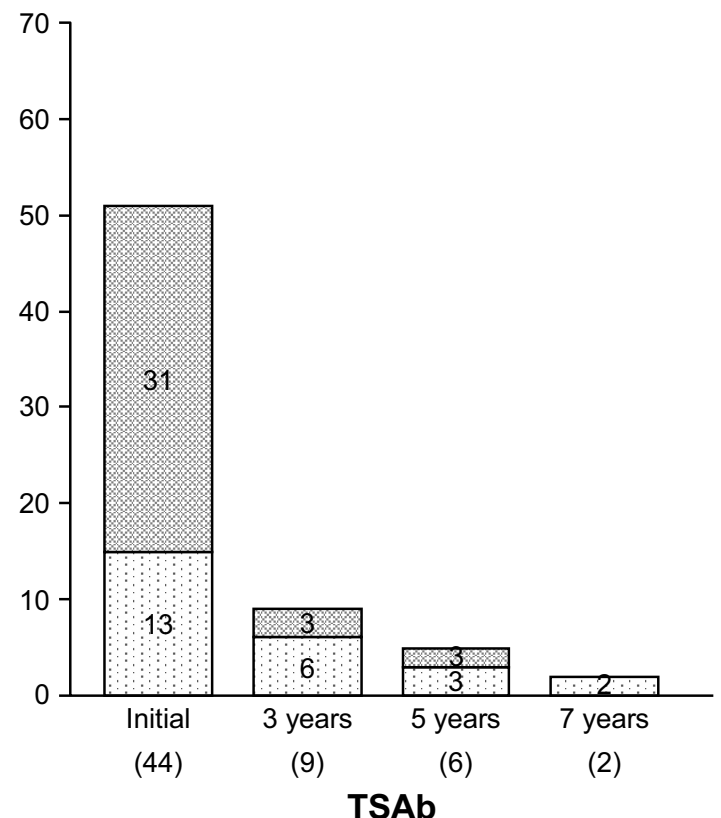

Positive $\$$ Negative

Figure 3 Transition of the TBII and TSAb positive rates in EGD patients.

Notes: Upon initial presentation, 74.5\% (4I/55) and 70.5\% (3I/44) of the patients with EGD proved to be TBII and TSAb positive, respectively. The positive rate for both TBII and TSAb gradually decreased spontaneously, with the TRAb becoming negative at 7 years after the initial development in all the patients tested. Although there was only a small number of patients who were measured for TRAb throughout the observation period, the thyroid function continued to be checked in these patients during the entire study period. Specifically, there were 33 patients at 3 years, 18 patients at 5 years and II patients at 7 years, all of whom exhibited a normal thyroid function.

Abbreviations: TBII, thyrotropin-binding inhibitory immunoglobulin; TSAb, thyroid-stimulating immunoglobulin; EGD, euthyroid Graves' disease; TRAb, thyrotropin receptor antibodies. 
Table 2 Itemized ophthalmic initial treatments in 58 EGD patients

\begin{tabular}{ll}
\hline Treatment & $\begin{array}{l}\text { Number of } \\
\text { patients (58) }\end{array}$ \\
\hline $\begin{array}{l}\text { With treatment } \\
\text { Monotherapy } \\
\text { Local injection of steroid }\end{array}$ & 29 \\
$\quad$ Surgery & II \\
$\quad$ Radiation & $\mathrm{I}$ \\
Combined therapy & 8 \\
$\quad$ Radiation + surgery & \\
$\quad$ Radiation + local injection of steroid & 2 \\
$\quad$ Radiation + local injection of steroid + surgery & 2 \\
$\quad$ Radiation + local injection of steroid + oral steroid & $\mathrm{I}$ \\
$\quad$ Surgery + local injection of steroid + oral steroid & $\mathrm{I}$ \\
Without treatment & 30
\end{tabular}

Notes: As additional treatments, intravenous steroid pulse therapy, local injection of steroid and surgery were administered to 3 patients, respectively. One of these patients did not need any treatment at the initial visit, but then steroid pulse therapy was needed 2 years after the development of EGD.

Abbreviation: EGD, euthyroid Graves' disease.

spontaneous improvement over time except in one case. Similarly, the number of the patients who were found to be positive for TBII and TSAb spontaneously decreased over time. Furthermore, among these 29 patients without eye treatments, $62.9 \%(17 / 27)$ and $54.5 \%(12 / 22)$ were positive for TBII and TSAb at their initial visit, while $18.2 \%(4 / 22)$ and $0 \%(0 / 12)$ were positive for TBII and TSAb at their last visit, respectively. Detailed observations were available for 18 patients out of the 29 patients. Figure 4 shows the course of the eye symptoms in these patients. Out of all the eye symptoms observed, only the value of the eye lid fissure showed a statistically significant improvement ( $p=0.0192)$. Comparisons of the TBII and TSAb values at the initial presentation among the patients with or without each of the treatments (local triamcinolone injection, radiotherapy and surgery) found no significant differences between the groups. Detailed $p$-values were as follows: local triamcinolone injection $p=0.096$, radiotherapy $p=0.8932$ and surgery $p=0.438$ for TBII and $p=0.4301, p=0.2995$ and $p=0.1190$ for TSAb, respectively. Furthermore, there was also no significant difference for the TBII and TSAb values between the 30 patients who required eye treatment and the 28 patients who did not $(p=0.2109,0.3434)$.

\section{Discussion}

In this retrospective study, $24.1 \%$ of the patients diagnosed with EGD at the initial presentation developed hyperthyroidism, while $3.4 \%$ developed permanent hypothyroidism. By contrast, Wiersinga et $\mathrm{al}^{11}$ found that 10 (55.6\%) out of 18 EGD patients developed hyperthyroidism and 3 (16.7\%) developed hypothyroidism. The reason for this variation in the results may be due to a difference in the

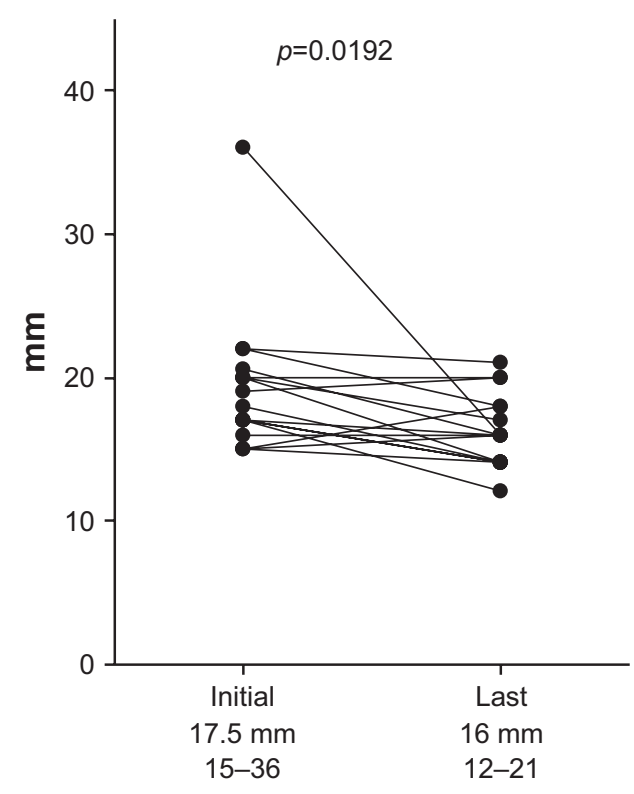

(18)

(18)

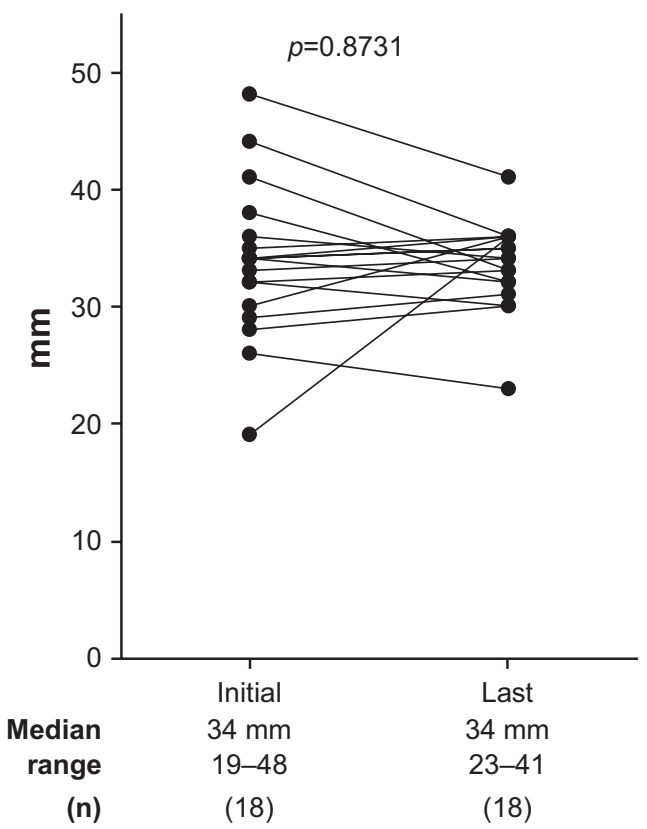

Proptosis $R+L$

Figure 4 Clinical course of ophthalmic symptoms in 18 EGD patients without any eye treatments.

Notes: We examined 18 out of the 28 EGD patients without eye treatments in detail. Median observation time was 4.I years (range I.7-II.9). In most patients, the items observed spontaneously improved over time. In particular, a significant difference was observed for the improvements in the eye lid fissure.

Abbreviations: EGD, euthyroid Graves' disease; L, left; R, right. 
number of subjects, the duration of the follow-up, the level of iodine intake or the genetic background.

According to 14 studies that examined EGD patients and were published between 1986 and 2016,,$^{6,11-23}$ the positive rates for the anti-thyroid antibodies were as follows: TBII $24.5 \%$ (35/143) and TSAb 64.2\% (79/123). By contrast, the values for the initial visit in our current study were as follows: TBII $74.5 \%$ (41/55) and TSAb 70.5\% (31/44). However, it should be noted that since it was not possible to detect TSH with a high sensitivity until 1995, it is possible that 5 out of the 14 studies could have included patients with subclinical thyroid dysfunction. Thus, this could be one reason for the difference in the positive rates for the antibodies that is seen between the previous reports and our current study. Moreover, Khoo et $\mathrm{al}^{5}$ used four different measurement kits to determine the TRAb values in 6 EGD patients. While this previous study did show there was a higher prevalence of TSAb positivity versus TBII in EGD patients, it also reported that changing the concentration of polyethylene glycol caused a fluctuation in the TSAb positivity. In addition, when the TBII values were compared between porcine and human kits, the positive rate was higher for the human kit in the same subjects. While this suggests that the difference in the measuring method could be related to the difference observed for the TSAb and TBII positivity, the number of subjects in the study was small, and thus, a further analysis with a larger number of subjects will need to be undertaken.

In the current study, $42(72.4 \%)$ out of the 58 EGD patients remained euthyroid, although they had a high prevalence of TBII and TSAb. The reason why EGD patients develop TAO without developing thyroid dysfunction is currently unknown. One hypothesis has proposed that the thyroid tissue of EGD patients has chronic lymphocytic thyroiditislike changes, with the tissue not able to react to the TRAb stimulus. ${ }^{22,24}$ However, others have pointed out there are some contradictions regarding this hypothesis. For example, in the subjects who were examined, the prevalence of the positivity for thyroglobulin antibody ( $\mathrm{TgAb}$ ) or thyroperoxidase antibody (TPOAb) was cited as being too low, while other pathological findings showed that there was only epithelial hyperplasia. ${ }^{25}$ Since the TRAb results were much more useful than either the TgAb or TPOAb when making the Graves' disease diagnosis, most of the subjects in our current study were not measured for $\mathrm{TgAb}$ and TPOAb.

Furthermore, another case report found that there was normal thyroid tissue in an EGD patient. ${ }^{26}$ In addition, another study hypothesized that the presence of TSAb could lead to the development of TAO, while the presence of a high titer of TSAb and TBII might result in the development of hyperthyroidism.22 As our current study did not find any differences for the positive rates of TSAb and TBII, it is our hypothesis that a different theory might explain these results. During the thyroid hormone synthesis in thyrocytes, there are two major known signaling pathways, with one process utilizing cyclic AMP (c-AMP) and the other utilizing diacylglycerol (DAG). In order to synthesize thyroid hormone, both of these pathways are essential, and thus, similar to TSH or TBII, stimulation of the TSH receptor activates both pathways. By contrast, TSAb is measured by the amount of c-AMP that is produced after the TSAb stimulus. However, the pathway that participates in the DAG pathway has yet to be currently evaluated. Therefore, if TSAb can only activate the c-AMP pathway, then synthesis of the thyroid hormone cannot proceed and may be the reason why EGD patients remain euthyroid. In addition, since there is no commercially available kit that can measure the activity of the DAG pathway, further investigations of this pathway are not yet possible. Additional analyses will need to be undertaken in order to definitively clarify these relationships.

The one limitation of our study was that we used a nonstandard method for evaluating the eye symptoms. Since the observation period for this study ranged from 1987 to 2017, the currently established and consistent methodologies that are now being used to evaluate ophthalmic symptoms, such as the Clinical Activity Score (CAS), were not available for use during our experimental period. Since CAS is the most frequently used and reliable method for evaluating the TAO activity, the use of CAS would have been a better way to evaluate the symptoms. However, Gerding et al did report finding a strong correlation between the CAS and both TBII and TSAb. ${ }^{27}$ Unfortunately, CAS was not developed until 1999, which was also the year that EUGOGO was founded. Furthermore, some of the subjects in our study were diagnosed prior to 1999. Thus, it was not possible to use CAS in all the patients that we evaluated. As a result, we chose to evaluate all the patients in our current study using proptosis, lid fissure and the cross-sectional area of the extraocular rectus muscles. In addition, since most patients did not show any evidence of clinical deterioration of their eye symptoms, there were only a few patients who required a follow-up with imaging evaluation. As a result, we could not collect enough cross-sectional data from the rectus muscles to make it possible to perform a proper statistical analysis.

\section{Conclusion}

EGD patients were positive for both TBII and TSAb in about $70 \%$ of the patients examined, with this frequency gradually 
decreasing and eye symptoms spontaneously improving over time. This study found no correlations between TRAb and eye symptoms. Thyroid functions in EGD patients who have been euthyroid for more than 6.7 years might possibly remain euthyroid in the future.

\section{Disclosure}

The authors report no conflicts of interest in this work.

\section{References}

1. Rundle FF, Wilson CW. Development and course of exophthalmos and ophthalmoplegia in Graves' disease with special reference to the effect of thyroidectomy. Clin Sci. 1945;5(3-4):177-194.

2. Burch HB, Wartofsky L. Graves' ophthalmopathy: current concepts regarding pathogenesis and management. Endocr Rev. 1993;14(6): 747-793.

3. Marcocci C, Bartalena L, Bogazzi F, Panicucci M, Pinchera A. Studies on the occurrence of ophthalmopathy in Graves' disease. Acta Endocrinol (Copenh). 1989;120(4):473-478.

4. Bartley GB, Fatourechi V, Kadrmas EF, et al. Clinical features of Graves' ophthalmopathy in an incidence cohort. Am J Ophthalmol. 1996; 121(3):284-290.

5. Khoo DH, Eng PH, Ho SC, et al. Graves' ophthalmopathy in the absence of elevated free thyroxine and triiodothyronine levels: prevalence, natural history, and thyrotropin receptor antibody levels. Thyroid. 2000;10(12):1093-1100.

6. Tabasum A, Khan I, Taylor P, Das G, Okosieme OE. Thyroid antibodynegative euthyroid Graves' ophthalmopathy. Endocrinol Diabetes Metab Case Rep. 2016;2016:160008.

7. Murakami Y, Kanamoto T, Tuboi T, Maeda T, Inoue Y. Evaluation of extraocular muscle enlargement in dysthyroid ophthalmopathy. Jpn J Ophthalmol. 2001;45(6):622-627.

8. Costa RM, Dumitrascu OM, Gordon LK. Orbital myositis: diagnosis and management. Curr Allergy Asthma Rep. 2009;9(4):316-323.

9. Sato S, Noh JY, Sato S, et al. Comparison of efficacy and adverse effects between methimazole $15 \mathrm{mg}+$ inorganic iodine $38 \mathrm{mg} /$ day and methimazole $30 \mathrm{mg}$ /day as initial therapy for Graves' disease patients with moderate to severe hyperthyroidism. Thyroid. 2015;25(1):43-50.

10. Prummel MF, Terwee $\mathrm{CB}$, Gerding MN, et al. A randomized controlled trial of orbital radiotherapy versus sham irradiation in patients with mild Graves' ophthalmopathy. J Clin Endocrinol Metab. 2004;89(1): 15-20.

11. Wiersinga WM, Smit T, van der Gaag R, Koornneef L. Temporal relationship between onset of Graves' ophthalmopathy and onset of thyroidal Graves' disease. J Endocrinol Invest. 1988;11(8):615-619.

12. Arslan E, Yavaşoğlu I, Cildağ BM, Kocatürk T. Unilateral optic neuropathy as the initial manifestation of euthyroid Graves' disease. Intern Med. 2009;48(22):1993-1994.
13. Peter S. Euthyroid Graves' ophthalmopathy in a child. West Indian Med J. 2006;55(5):360.

14. Cakir M. Euthyroid Graves' ophthalmopathy with negative autoantibodies. J Natl Med Assoc. 2005;97(11):1547-1549.

15. Kubo T, Toki J, Kado Y, et al. Thyroid-stimulating antibody in a patient with euthyroid Graves' disease. Endocr J. 2000;47(2):197-201.

16. Hamada N, Okamoto Y, Yoshida H, Tsumura K, Nakamura Y, Noh JY. Sympathetic overactivity in the development of eyelid retraction in a patient with euthyroid Graves' disease evaluated by accommodation. Endocr J. 2000;47(5):623-628.

17. Kazuo K, Fujikado T, Ohmi G, Hosohata J, Tano Y. Value of thyroid stimulating antibody in the diagnosis of thyroid associated ophthalmopathy of euthyroid patients. Br J Ophthalmol. 1997;81(12):1080-1083.

18. Watanabe M, Iwatani Y, Kashiwai T, Iijima T, Fujikado T, Amino N. Euthyroid Graves' disease showing no thyroid abnormalities except positive thyroid-stimulating antibody (TSAb): two case reports. $J$ Intern Med. 1995;238(4):379-384.

19. Kashiwai T, Tada H, Asahi K, et al. Significance of thyroid stimulating antibody and long term follow up in patients with euthyroid Graves' disease. Endocr J. 1995;42(3):405-412.

20. Kosugi S, Inoue D, Sugawa H, Enomoto T, Mori T, Imura H. Similarity and dissimilarity between clinical and laboratory findings, especially anti-thyrotropin receptor antibody in ophthalmic Graves' disease without persistent hyperthyroidism and hyperthyroid Graves' disease. Endocrinol Jpn. 1990;37(3):343-354.

21. Uno C, Nishikawa M. [Clinical studies on abnormal thyroid stimulators in patients with Graves' disease. II. Clinical significance of measuring TSAb and TBII in patients with euthyroid Graves' disease and patients with hyperthyroid Graves' disease during antithyroid drug treatment]. Nihon Naibunpi Gakkai Zasshi. 1988;64(3):206-215. Japanese [with English abstract].

22. Kasagi K, Hatabu H, Tokuda Y, Iida Y, Endo K, Konishi J. Studies on thyrotrophin receptor antibodies in patients with euthyroid Graves' disease. Clin Endocrinol (Oxf). 1988;29(4):357-366.

23. Peter SA. Euthyroid Graves' disease. Report of a case observed over a 12-year period. Am J Med. 1986;80(6):1197-1198.

24. Tamai H, Ohsako N, Takeno K, et al. Changes in thyroid function in euthyroid subjects with a family history of Graves' disease: a follow-up study of 69 patients. J Clin Endocrinol Metab. 1980;51(5): 1123-1127.

25. Kasagi K, Hatabu H, Miyamoto S, et al. Scintigraphic findings of the thyroid in hypothyroid patients with blocking-type TSH-receptor antibodies. Eur J Nucl Med. 1994;21(9):962-967.

26. Suzuki N, Yoshihara A, Yoshimura Noh J, et al. Pathological findings of the thyroid tissue in a patient with euthyroid Graves' disease. Intern Med. 2015;54(22):2881-2884.

27. Gerding MN, van der Meer JW, Broenink M, Bakker O, Wiersinga WM, Prummel MF. Association of thyrotrophin receptor antibodies with the clinical features of Graves' ophthalmopathy. Clin Endocrinol $(O x f)$. 2000; 52(3):267-271.
Clinical Ophthalmology

\section{Publish your work in this journal}

Clinical Ophthalmology is an international, peer-reviewed journal covering all subspecialties within ophthalmology. Key topics include: Optometry; Visual science; Pharmacology and drug therapy in eye diseases; Basic Sciences; Primary and Secondary eye care; Patient Safety and Quality of Care Improvements. This journal is indexed on Submit your manuscript here: http://www.dovepress.com/clinical-ophthalmology-journal
Dovepress

PubMed Central and CAS, and is the official journal of The Society of Clinical Ophthalmology (SCO). The manuscript management system is completely online and includes a very quick and fair peer-review system, which is all easy to use. Visit http://www.dovepress.com/ testimonials.php to read real quotes from published authors. 